\title{
Separate muscle bundles of the flexor digitorum superficialis overlying the ulnar nerve
}

\author{
D.-K. Han, H.-S. Won, H.-F. Liu, I.-H. Chung, I.-B. Kim \\ Catholic Institute for Applied Anatomy, Department of Anatomy, The Catholic University of Korea, Seoul, Republic of Korea
}

[Received 23 December 2014; Accepted 3 April 2015]

Background: The aim of this study was to elucidate the morphological characteristics of the muscle bundles of the flexor digitorum superficialis (FDS) attached to the intermuscular aponeurosis (IMA) and any related structure that could potentially compress the ulnar nerve.

Materials and methods: Fifty embalmed limbs of 34 adult cadavers were studied. Results: The FDS arose as multiple separate bundles from the IMA of the lateral surface of the flexor carpi ulnaris in $76 \%$ of specimens. Below their origin, these separate bundles became attached continuously as a single mass to form the muscle belly. There were 1, 2, 3, 4 and 5 arising FDS muscle bundles in 28\%, $30 \%, 4 \%, 10 \%$ and $4 \%$ of specimens, respectively. The muscle bundles were attached either only superficially (24\% of cases) or across the entire width $(20 \%$ of cases) of the IMA. In 32\% of the specimens, bundles arose from the IMA in a combined fashion, being attached to the IMA superficially, deep and across the entire structure. The muscle bundles that arose from the deep part or entire width of the IMA were in contact with the ulnar nerve in 52\% of specimens. In $11(22 \%)$ specimens, the deep borders of the lowest muscle bundles close to the ulnar nerve were composed of tendinous fibres that divided from the IMA of the lateral surface of the flexor carpi ulnaris. The distance from the medial epicondyle to the lowest point of the FDS arising from the IMA was $62.0 \pm 19.7 \mathrm{~mm}$.

Conclusions: The thick tendinous deep border of the lowest muscle bundle of the FDS where it attaches to the IMA is a potential cause of ulnar nerve compression. (Folia Morphol 2015; 74, 4: 434-438)

Key words: flexor digitorum superficialis, ulnar nerve, compression, intermuscular aponeurosis

\section{INTRODUCTION}

The humeroulnar head of the flexor digitorum superficialis (FDS) arises from the medial epicondyle, the ulnar collateral ligament, the adjacent intermuscular septa and the coronoid process $[4,18,19]$. The muscular parts originating from the intermuscular septum, which comprises the aponeurosis between the FDS and the flexor carpi ulnaris (FCU), appear as a single mass, but when pulling the FCU medially, the lower part of the FDS usually forms separate muscle bundles.

The ulnar nerve enters the forearm between the two heads of origin of the FCU and descends to the flexor digitorum profundus, covered by the FCU in the upper part of the forearm $[4,19,23]$. In the lower forearm, the ulnar nerve rests on the flexor digitorum profundus between the FCU and the FDS [3]. Ulnar nerve compression occurs commonly in the 


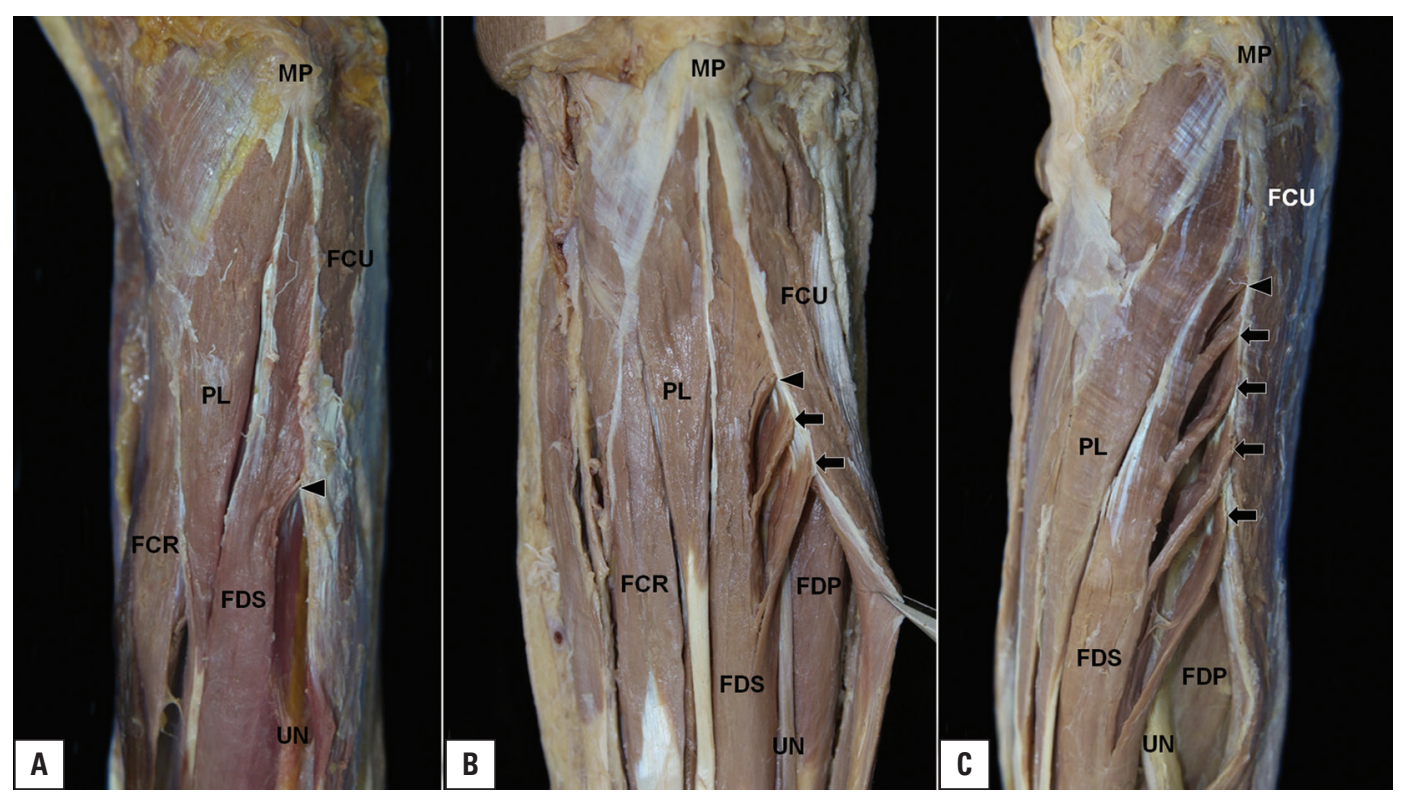

Figure 1. The flexor digitorum superficialis (FDS) originating from the intermuscular aponeurosis (IMA). The FDS is shown arising as a single, continuous muscle belly mass (A), and as two (B) and four (C) muscle bundles attached to the IMA. Arrowheads indicate the lower border of the FDS arising from the IMA as a continuous muscle belly. Arrows indicate the separate muscle bundles of the FDS from the IMA; FCU — flexor carpi ulnaris; FCR — flexor carpi radialis; FDP — flexor digitorum profundus; MP — medial epicondyle; PL — palmaris longus; UN — ulnar nerve.

cubital tunnel, but also (although rarely) in the region of the upper forearm below the cubital tunnel [9]. The structures thought to be responsible for ulnar nerve compression below the cubital tunnel are the muscular fascia under the FCU [20] and the intermuscular aponeurosis (IMA) between the FCU and the FDS $[2,5]$. The muscle bundles of the FDS attached to the IMA between the FCU and the FDS reportedly create a muscle tunnel over the ulnar nerve [13]. The muscle bundle overlying the ulnar nerve may cause compressive neuropathy [7, 22].

The aim of this study was to elucidate the morphological characteristics of the muscle bundles of the FDS attached to the IMA and any related structure that could potentially compress the ulnar nerve.

\section{MATERIALS AND METHODS}

Fifty embalmed limbs of 34 adult cadavers (19 males, 15 females; mean age, 79.7 years; age range, 46-94 years) were studied. Both sides of 16 of these cadavers were used, and the same numbers of right and left sides were examined $(n=25)$. The FDS and FCU muscles were exposed after removing the antebrachial fascia, and the parts of the FDS attached to the IMA that pull the FCU medially were examined. All distances were measured as vertical lines from the medial epicondyle of the humerus. Chi-square test was used to compare the incidences related with the morphologic variations of the separate bundles of FDS between two genders and between left and right sides, with a statistical significance level of $p<0.05$.

\section{RESULTS}

The FDS arose as multiple separate bundles from the IMA of the lateral surface of the FCU in $76 \%$ of specimens; the bundles merged below their origin to become a single mass, forming the muscle belly (Fig. 1). The number of FDS muscle bundles varied from 1 to 5 , with $1,2,3,4$ and 5 bundles being found in $28 \%$, $30 \%, 4 \%, 10 \%$ and $4 \%$ of specimens, respectively. The muscle bundles arose either superficially (total $24 \%$; male $16 \%$; female $8 \%$ ) or across the entire width of the IMA (total 20\%; male 16\%; female 4\%; Fig. 2). In 32\% of the specimens, the bundles arose from the IMA in a combined fashion: superficially and across the entire IMA (total $8 \%$; male $6 \%$; female $2 \%$ ), superficially and deep (only female $6 \%$ ), deep and across the entire IMA (total $10 \%$; male $8 \%$; female $2 \%$ ), and superficially, deep and across the entire IMA (total $8 \%$; male $2 \%$; female $6 \%$ ). The muscle bundles that arose from the deep part or entire width of the IMA were in contact with the ulnar nerve ( $52 \%$ of cases; Figs. 2,3$)$. The deep borders of the proximal muscle bundles consisted of the fleshy muscle belly, whereas those of the lowest muscle 


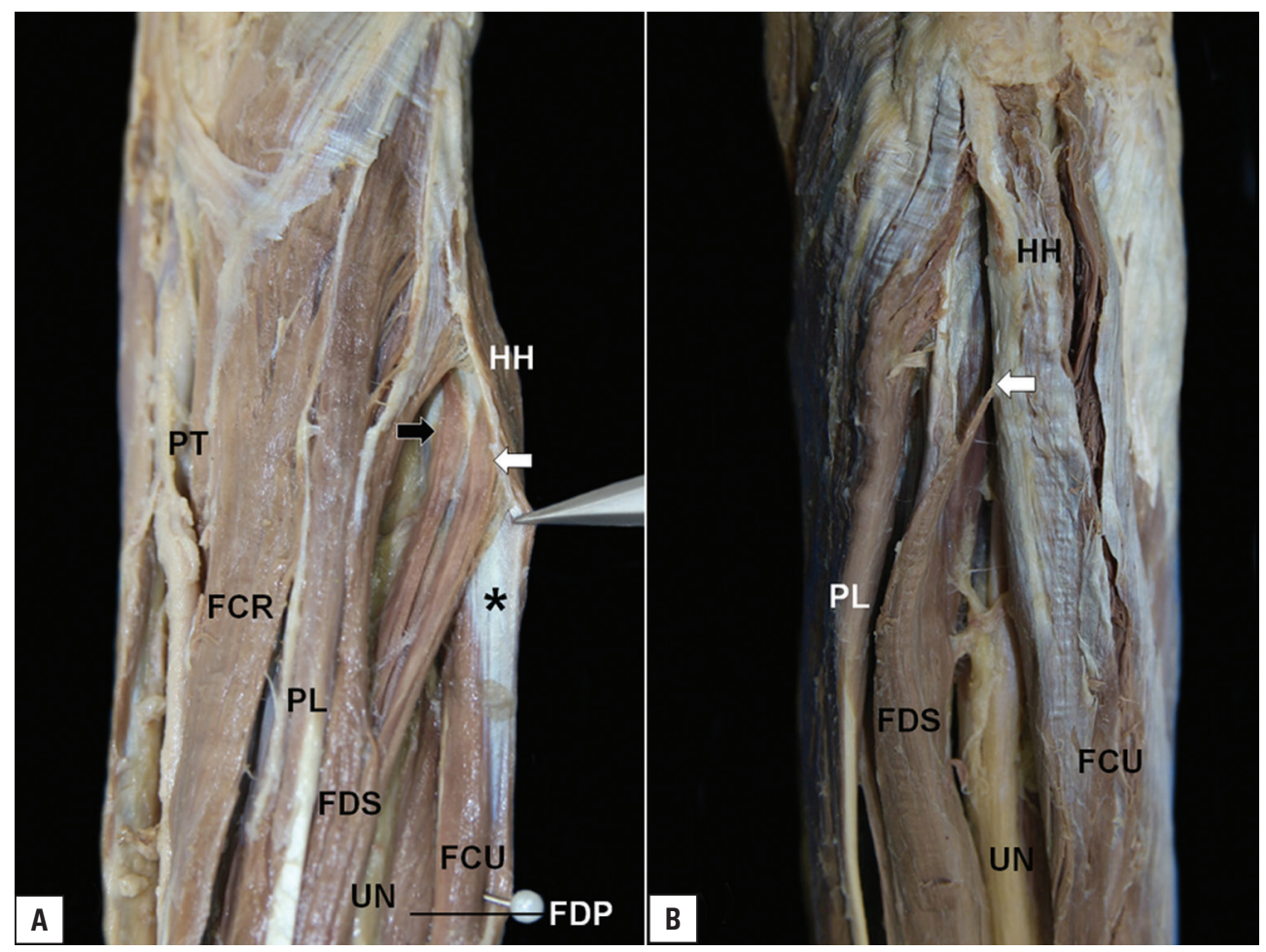

Figure 2. The muscle bundles of the flexor digitorum superficialis (FDS) attached to different parts of the intermuscular aponeurosis (IMA); A. Two bundles of the FDS attached separately to the deep (black arrow) and superficial (white arrow) parts of the IMA; B. The muscle bundle in this specimen is attached only to the superficial part of the IMA. The asterisk indicates the IMA; HH — humeral head of the flexor carpi ulnaris; PT — pronator teres; rest abbreviations as in Figure 1.

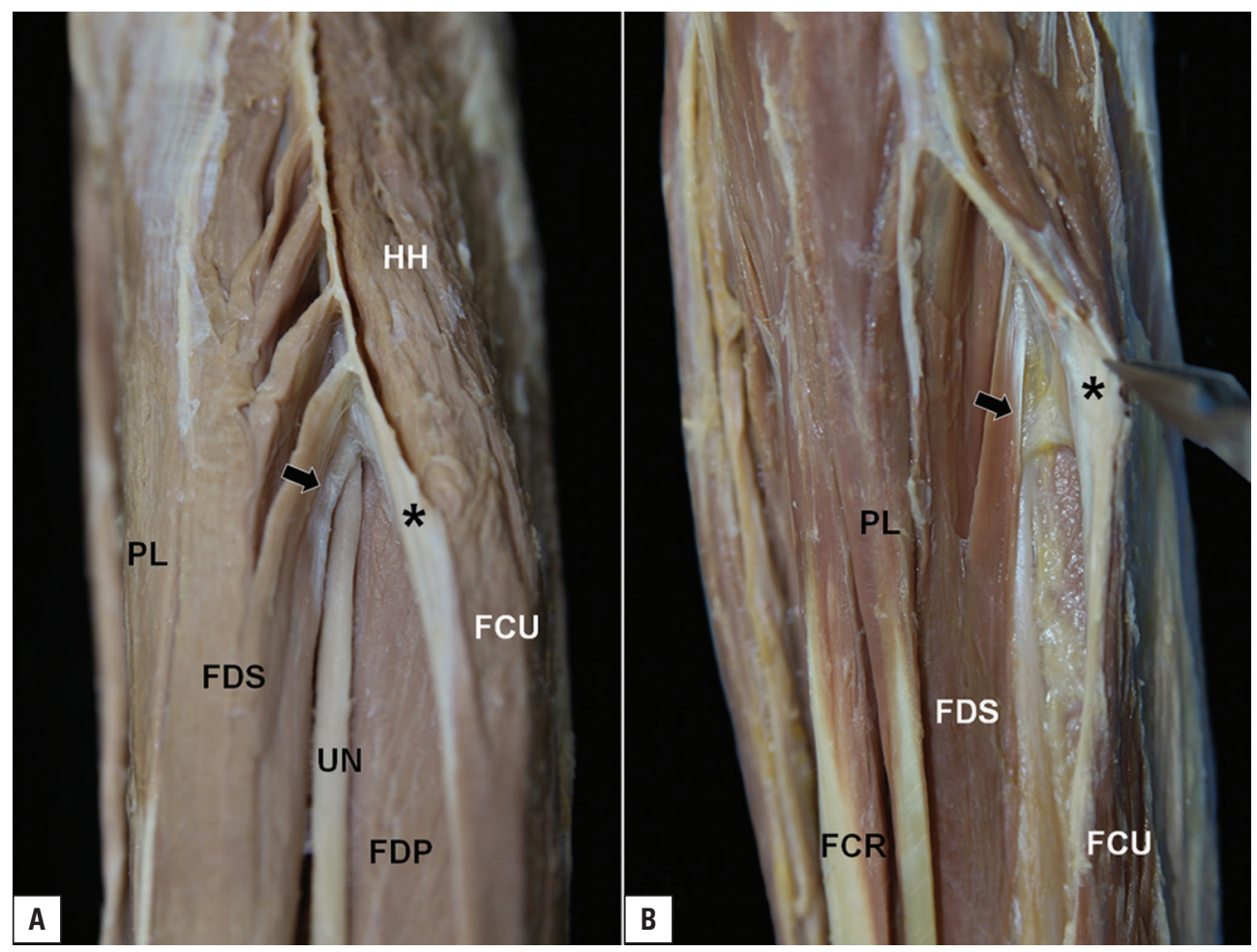

Figure 3. Tendinous fibres of the deep border of the flexor digitorum superficialis muscle bundle. The tendinous deep border (arrows) of the lowest bundle is observed attached across the entire (A) and deep (B) parts of the intermuscular aponeurosis (asterisks), crossed over the ulnar nerve; PT — pronator teres; abbreviations as in Figures 1 and 2. 


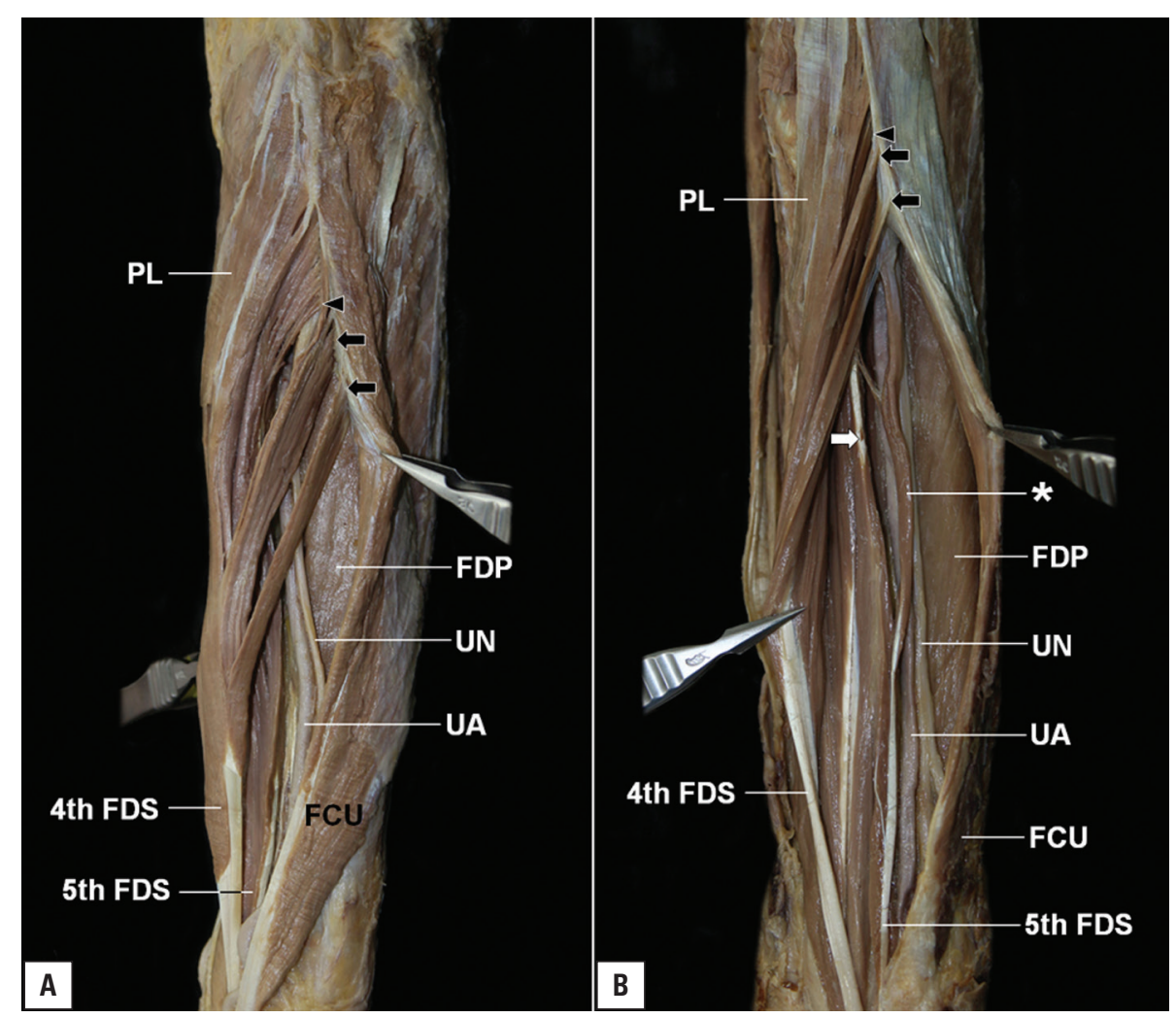

Figure 4. The flexor digitorum superficialis (FDS) muscle bundles continued to the ring finger tendon ( $\mathbf{A}$ ) and to the little finger (B, asterisk). Arrowheads indicate the lower border of the FDS arising from the intermuscular aponeurosis (IMA) as a continuous muscle belly. Black arrows indicate the separate muscle bundles of the FDS from the IMA. The white arrow indicates the intermediate tendon; UA — ulnar artery; rest abbreviations as in Figure 1.

bundles close to the ulnar nerve were composed of the tendinous fibres that were divided from the IMA of the lateral surface of the FCU ( $n=11$ specimens, $22 \%$; Fig. 3). These tendinous fibres were observed mainly in the bundles arising across the entire width of the IMA (16\%), but in rare cases were also found in those arising from the deep (4\%) and superficial $(2 \%)$ parts of the IMA. And these tendinous fibres were more frequently observed in right side than in left side, which were statistically significant $(p<0.05)$. All except one of the separate muscle bundles from the IMA continued to the ring finger (Fig. 4). In one specimen, the lowest muscle bundle formed the proximal belly of the little finger, and joined the distal belly that originated from the intermediate tendon (Fig. 4). The distances between the medial epicondyle of the humerus to the styloid process of the ulna and to the lowest point of the FDS arising from the IMA were $241.8 \pm 15.8 \mathrm{~mm}$ (mean \pm \pm standard deviation) and $62.0 \pm 19.7 \mathrm{~mm}$, respectively.

\section{DISCUSSION}

Multiple muscle bundles of the FDS arising from the IMA between the FDS and FCU have been reported previously in 4 of 12 limbs, with these bundles overlying the ulnar nerve [13]. In the present study, there were between 1 and 5 separate FDS bundles in $76 \%$ of 50 limbs. Among these, the muscle bundles were overlying the ulnar nerve in $52 \%$ of the specimens. In all except 1 case, these bundles continued to the tendon of the ring finger (Fig. 4); in 1 limb, the FDS muscle belly of the little finger originated from the intermediate tendon attached to the medial epicondyle [1], and the belly attached to the IMA that crossed the ulnar nerve obliquely.

The muscles thought to be associated with ulnar nerve compression have been the hypertrophied medial head of the triceps brachii [17], and anomalous muscles such as the anconeus epitrochlearis and the anconeus epimedialis muscles $[6,8,15]$ at the elbow, and a hypertrophied FCU in the lower part of the forearm [11]. Nonhypertrophied anomalous muscle bands that crossed the ulnar nerve were also found to cause compressive neuropathy around the wrist $[16,22]$. It has been suggested that these anomalous muscle bands found in cadavers could compress the ulnar nerve in vivo $[7,12]$. 
Nerve compression occurs when pressure is exerted on a nerve trapped between unyielding structures in fibro-osseous canals such as the cubital or ulnar tunnel [14]. Compressive neuropathies caused by muscle may be attributable to hypertrophy of the normal muscle, crossing of a muscle band over a nerve, and the tendinous band from normal or anomalous muscles. Nerve compression thus appears to be due to several factors, including the size of the anomalous muscle, its oblique course, its thickened posterior fascia and the spatial constraints imposed by the underlying bone [21].

The muscle bundles of the FDS attached to the deep or entire width of the IMA between the FDS and FCU crossed obliquely and were contact with the ulnar nerve on the flexor digitorum profundus muscle in some specimens (Fig. 3). The deep border of the lowest bundle was composed of the tendinous fibres in $22 \%$ and was closely contiguous with the ulnar nerve. It has been proposed that the IMA between the FDS and FCU could cause persistent symptoms following surgical release in cubital tunnel syndrome, and be the site of ulnar neuropathy at the elbow $[2,5,10]$.

\section{CONCLUSIONS}

The thick tendinous deep border of the lowest muscle bundle of the FDS attached to the IMA may potentially compress the ulnar nerve.

\section{REFERENCES}

1. Agee J, McCarroll HR, Hollister A (1991) The anatomy of the flexor digitorum superficialis relevant to tendon transfers. J Hand Surg Br, 16: 68-68.

2. Amadio PC, Beckenbaugh RD (1986) Entrapment of the ulnar nerve by the deep flexor-pronator aponeurosis. J Hand Surg Am, 11: 83-87.

3. Anson BJ (1966) Morris' human anatomy, 12th Ed. McGraw-Hill, New York.

4. Berry MM, Standring SM, Bannister LH (1995) Nervous system. In: Williams PL ed. Gray's anatomy, 38th Ed. Churchill Livingstone, Edinburgh, pp. 901-1398.

5. Campbell WW, Pridgeon RM, Riaz G, Astruc J, Sahni KS (1991) Variations in anatomy of the ulnar nerve at the cubital tunnel: pitfalls in the diagnosis of ulnar neuropathy at the elbow. Muscle Nerve, 14: 733-738.

6. Chalmers J (1978) Unusual causes of peripheral nerve compression. Hand, 10: 168-175.
7. Claassen H, Schmitt O, Schulze M, Wree A (2013) Variation in the hypothenar muscles and its impact on the ulnar tunnel syndrome. Surg Radiol Anat, 35: 893-899.

8. Dellon AL (1986) Musculotendinous variations about the medial humeral epicondyle. J Hand Surg Br, 11: 175-181.

9. Gabel GT, Amadio PC (1990) Reoperation for failed decompression of the ulnar nerve in the region of the elbow. J Bone Joint Surg Am, 72: 213-219.

10. Green JR, Rayan GM (1999) The cubital tunnel: anatomic, histologic, and biomechanical study. J Shoulder Elbow Surg, 8: 466-470.

11. Harrelson JM, Newman M (1975) Hypertrophy of the flexor carpi ulnaris as a cause of ulnar-nerve compression in the distal part of the forearm. J Bone Joint Surg Am, 57: 554-555.

12. Hill ML, Shoja MM, Salter EG, Tubbs RS (2006) An unusual muscle of the wrist with potential compression of the ulnar nerve. Folia Morphol, 65: 178-180.

13. Karatas A, Apaydin N, Uz A, Tubbs SR, Loukas M, Gezen F (2009) Regional anatomic structures of the elbow may potentially compress the ulnar nerve. J Shoulder Elbow Surg, 18: 627-631.

14. Lahey MD, Aulicino PL (1986) Anomalous muscles associated with compression neuropathies. Orthop Rev, 15: 119-208.

15. Lee HJ, Kim JY, Jeon IH (2013) Cubital tunnel syndrome due to the anconeus epimedialis muscle. J Shoulder Elbow Surg, 22: e20-e22.

16. Luethke R, Dellon AL (1992) Accessory abductor digiti minimi muscle originating proximal to the wrist causing symptomatic ulnar nerve compression. Ann Plast Surg, 28: 307-308.

17. O'Hara JJ, Stone JH (1996) Ulnar nerve compression at the elbow caused by a prominent medial head of the triceps and an anconeus epitrochlearis muscle. J Hand Surg $\mathrm{Br}$, 21: 133-135.

18. Rouvière H, Delmas A (1991) Anatomie Humaine. Descriptive, Topographique et Fonctionnelle. Vol. 3, 13th Ed. Masson, Paris.

19. Salmons S (1995) Muscle. In: Williams PL ed. Gray's anatomy, 38th Ed. Churchill Livingstone, Edinburgh, pp. 737-900.

20. Siemionow M, Agaoglu G, Hoffmann R (2007) Anatomic characteristics of a fascia and its bands overlying the ulnar nerve in the proximal forearm: a cadaveric study. J Hand Surg Eur Vol, 32: 302-307.

21. Spinner RJ, Spinner M (1996) Superficial radial nerve compression at the elbow due to an accessory brachioradialis muscle: a case report. J Hand Surg Am, 21: 369-372.

22. Turner MS, Caird DM (1977) Anomalous muscles and ulnar nerve compression at the wrist. Hand, 9: 140-142.

23. Woodburne RT, Burkel WE (1988) Essentials of human anatomy. 8th Ed. Oxford University Press, New York. 